Plant Sciences Research 11 (2-4): 8-12, 2019

ISSN: 1995-476X

(C) Medwell Journals, 2019

\title{
Estimates of Heterosis Parameters for Starch Viscosity Properties in Rice (Oryza sativa L.)
}

\author{
${ }^{1}$ Alireza Haghighi Hasanalideh, ${ }^{2}$ Ezatollah Farshadfar and ${ }^{3}$ Mehrzad Allahgholipour \\ ${ }^{1}$ Young Researchers and Elite Club, Rasht Branch, Islamic Azad University, Rasht, Iran \\ ${ }^{2}$ Department of Production Engineering and Plant Genetics, Faculty of Agricultural \\ Sciences and Engineering, Campus of Agriculture and Natural Resources, \\ Razi University, Kermanshah, Iran \\ ${ }^{3}$ Department of Seed Improvement, Rice Research Institute of Iran, \\ Agricultural Research, Education and Extension Organization, Rasht, Iran
}

\begin{abstract}
This study was undertaken to assess heterosis and heterosis parameters of viscosity characters in rice with the aim of evaluating the using of hybrid base methods for improving rice cooking quality. $F_{2}$ progenies, from a $6 \times 6$ half diallel with their parents planted in a complete randomized block design with three replications at Rice Research Institute of Iran in 2015. The analysis of variance of RVA parameters through Gardner-Eberhart II method showed that mean square of genotype was significant for all viscosity characters. Also, mean square of variety and heterosis were significant for all studied traits. Average heterosis was significant for PV, FV and SV. Variety heterosis and specific heterosis were significant for traits PV, BV, FV and SV. Estimation of the variety effect $\left(\mathrm{v}_{\mathrm{i}}\right)$ and heterosis of varieties $\left(\mathrm{h}_{\mathrm{i}}\right)$ for viscosity characters revealed that the parent Line 23 had the most contribution to heterosis for PV, BV and FV. Use of this parent in hybrid base breeding methods will be promising to improving these traits. The highest estimate of $\mathrm{v}_{\mathrm{i}}$ observed in the parent RI18430-46 for PV and FV, parent Deylamani for BV and parent IR50 for SV. Therefore, use of these parents in selection base methods to improving these traits is promising. Results of relative heterosis through mid-parents and heterobeltiosis for viscosity characters in rice crosses revealed that heterobeltiosis for peak viscosity, breakdown viscosity, final viscosity and setback viscosity had a range of $-14.39 \%$ to $13.10 \%$, $-57.01 \%$ to $59.86 \%,-13.41 \%$ to $8.85 \%$ and $-22.45 \%$ to $29.10 \%$, respectively.
\end{abstract}

Key words: Diallel, heterobeltiosis, variety effect, heterosis effect, rice quality, RVA parameters

\section{INTRODUCTION}

Rice (Oryza sativa L.) is the staple food of many countries and is increasing in popularity in many other parts of the world. Consumers pay attention to rice grain quality, displaying a preference for rice with a particular visual appearance, texture, flavor and aroma (Fitzgerald et al., 2009). Crop quality is a key determinant of the economic and nutritional value of agricultural products, since, it directly affects acceptability to buyers and consumers (Koutroubas et al., 2004). Indeed, the achievement of superior quality standards allows farmers to gain a competitive market advantage with respect to imported low price products (Griglione et al., 2015). The texture of cooked rice is considered by many consumers to be a quality trait of primary importance. Texture is a complex trait perceived by humans and several instrumental tests are used to predict these sensory attributes or textural properties of rice. These tests involve assays that measure the physicochemical properties of rice such as alkali spreading value, gel consistency, Rapid
Visco Analyser (RVA) and texture analyser (Balindong et al., 2018). Methods currently in use to determine the biophysical properties of starch include instrumental techniques such as differential scanning calorimetry and the RVA. The RVA is using to determine viscosity changes associated with starch gelatinization and liquefaction including the effects of proteolysis and saccharification. Information related to the water-holding capacity of the starch or mixture during the analysis is provided by the Peak Viscosity (PV) and this is often correlated with different quality parameters of the sample. Other parameters calculated such as the rate of Breakdown in Viscosity (BV) to a holding strength, Hot Paste Viscosity (HPV) or Trough Viscosity (TV), depend on the temperature and degree of mixing or shear stress, applied to the mixture and the intrinsic properties of the sample analysed. The re-association between starch molecules during cooling is commonly referred to as the setback (SV). It involves retrogradation or re-ordering of the starch molecules and has been correlated with the texture of various products. The Final Viscosity (FV) is

Corresponding Author: Alireza Haghighi Hasanalideh, Young Researchers and Elite Club, Rasht Branch, Islamic Azad University, Rasht, Iran, Mobile No: +98 9330825955 
the parameter which is most commonly used to define the pasting properties of a given sample. The FV indicates the ability of the material to form a viscous paste or gel after cooking and cooling (Cozzolino, 2016). Pre-breeding allows plant breeders to increase frequency of favorable alleles from genetically diverse germplasm. This concept encompasses germplasm introduction, adaptation, evolution and improvement for breeding purposes. Heterosis is the natural phenomenon through which the hybrid progeny displays genetically improved traits in relation to its parents and it has been utilized in agriculture for the development of cultivars with better performance (Fu et al., 2014). Heterosis or hybrid vigor is the manifestation of the beneficial effects of hybridization, i.e., when the performance of a hybrid is superior to the average performance of its parents (Falconer, 1987). Mating designs have been widely used to examine the genetic components of the set of genotypes in terms of the genetic effects involved in quantitative traits. These designs allow screening of large population samples. The diallel analysis can provide estimates for genetic effects and a basis for the preliminary assessment of heterotic groups. The heterotic groups can be assumed based on their combining ability with other populations. Consequently, alternative heterotic patterns among population crosses can also be identified (Laude and Carena, 2014). The dial el analysis provides a systematic approach for identification of superior parents and crosses which is the basic material on which the success of a breeding programme rests. Exploitation of hybrid vigour has been recognized as an important tool for genetic improvement of yield and may serve as a major fruitful technique to break existing yield barriers, to get better transgressive segregants. Economic heterosis has the most parctical utility which would be compared with a standard variety as check has relatively limited importance and is of more academic interest than that of parctical utility. In practical plant breeding, the heterosis measured over better parent and popular cultivar is more realistic and is of more practical importance (Chauhan et al., 2016). Gardner and Eberhart (1966) proposed a model to estimate genetic effects from a diallel cross of a fixed set of random mating populations with arbitrary gene frequencies at all loci assuming diploid inheritance and no epistasis. Gardner-Eberhart Analysis II partitions the variation among all populations into varieties (parents) and mid-parent heterosis and the latter is subdivided into average heterosis $\bar{h}$ ) variety heterosis $\left(h_{i}\right)$ and specific heterosis $\left(\mathrm{s}_{\mathrm{ij}}\right)$. Relative to Gardner-Eberhart Analysis III, the Gardner-Eberhart Analysis II provides more information in terms of the number of genetic effects estimated. The overall heterosis $\left(h_{i j}\right)$ is subdivided to $\bar{h}$, $\mathrm{h}_{\mathrm{i}}$ and $\mathrm{s}_{\mathrm{ij}}$ which provide detailed explanation on the cause of heterosis. As explained by Gardner and Eberhart (1966), the $\bar{h}$ contributed by the parents used in crosses is the difference between the mean performance of all crosses and all parents. The $h_{i}$ is the mean heterosis contributed by parent $i$ in its crosses measured as the deviation from $\overline{\mathrm{h}}$. The $\mathrm{s}_{\mathrm{ij}}$ is the heterosis observed when parents $\mathrm{i}$ and $\mathrm{j}$ are mated (Laude and Carena, 2014). Jin et al. (2004) reported that the total main genetic variances accounted for over $64 \%$ of the total genetic variance for breakdown viscosity, consistency and setback, indicating that these traits were mainly controlled by the main genetic effects in addition to the genotype environment interaction effects. Jin et al. (2004) analyzed taste meter value and Starch RVA properties by Griffing's diallel method with eight indica rice cultivars. They revealed that the additive variation was principal in taste meter value, peak viscosity, trough viscosity, final viscosity, breakdown, setback, consistency while the non-additive variation was dominant in pasting temperature. Wu et al. (2002) reported presence of heterosis and difference in mean for hybrids and their parents in viscosity characters. Liu (2013) by using RVA to evaluate the heterosis of rice starch viscosity properties, reported highly significant heterosis over high-parent in peak viscosity and highly significant heterosis over mid-patent for trough viscosity, breakdown, final viscosity and consistency. The result of this study also, revealed highly significant negative heterosis over mid-parent and positive heterosis over low-parent in both setback and peak time. Moreover, highly significant negative heterosis over low-parent reported for pasting temperature. Considering the above contents, the present study was undertaken to assess heterosis and heterosis parameters of viscosity characters in rice with the aim of evaluating the using of hybrid base methods for improving rice cooking quality.

\section{MATERIALS AND METHODS}

The plant material consisted of six parent diallel cross excluding reciprocals. The experiment was conducted at Rice Research Institute of Iran (RRII) during 2012-2015. Six Iranian and foreign rice varieties (Table 1) were selected as parental lines in this study. These parents had differences in origin, pedigree and some of the quality and morphological traits (Table 1). Parent lines selected among 94 rice genotypes from different regions and origins thorough molecular analyzing for genetic diversity (Allhgholipour et al., 2014). These parental lines crossed in a diallel mating design in 2013. In order to produce $F_{2}$ progenies, $F_{1}$ populations from a $6 \times 6$ half diallel cross selfed in 2014 . The plant genetic materials (parents and $\mathrm{F}_{2} \mathrm{sec}$ ) planted in a complete randomized block design with three replications at RRII in 2015. After processing paddy to white rice, the samples were floured with a UD milling machine at a rate of 100 mesh. The rice flour samples 
Plant Sci. Res., 11 (2-4): 8-12, 2019

Table 1: Name, origin of country and pedigree of rice genotypes used in this study

\begin{tabular}{lll}
\hline Genotypes & Pedigree & Origin \\
\hline Gilaneh $^{\mathrm{a}}$ & Saleh/Abjiboji $/ /$ Abjiboji & RRII $^{\mathrm{c}}$, Iran \\
Daylamani & Local cultivar & Mazandran, Iran $^{\mathrm{b}}$ \\
IR50 & IR50 & IRRI $^{\mathrm{d}}$, Philippines \\
Line 23 & IR75479-199-3-3 & IRRI, Philippines \\
RI18447-2 & Sepidrood $/$ Gharib $^{\mathrm{b}}$ & RRII, Iran \\
RI18430-46 & Saleh $^{\mathrm{a}} /$ Hashemi $^{\mathrm{b}}$ & RRII, Iran \\
\hline
\end{tabular}

${ }^{\mathrm{a}}$ Improved rice variety of Iran, ${ }^{\mathrm{b}} \mathrm{Local}$ rice variety of Iran, ${ }^{\mathrm{c}}$ Rice research institute of Iran, ${ }^{\mathrm{d}}$ International rice research

(3g, 14\% moisture) were slurried with distilled water $(25 \mathrm{~mL})$ in an aluminum vessel. The pasting properties were measured by an RVA (RVA-3D Model, Newport Scientific, Sydney, Australia). A rice standard profile was used and the heating and cooling cycle was as follows: the starting temperature was $50^{\circ} \mathrm{C}$ which was held for $1 \mathrm{~min}$ then the sample was heated through linear increasing of temperature to $95^{\circ} \mathrm{C}$ in 4 min and $48 \mathrm{sec}$ and this was maintained for $7 \mathrm{~min}$ and $18 \mathrm{sec}$ finally, the sample was cooled to $50^{\circ} \mathrm{C}$ (linear decreasing in temperature for 11 min and $6 \mathrm{sec}$ ) which was held for $12 \mathrm{~min}$. The device was set to start at $960 \mathrm{rpm}$ in the first $10 \mathrm{sec}$ and after, the speed was set at $160 \mathrm{rpm}$. Then starch RVA parameters including Peak Viscosity (PV), Breakdown Viscosity (BV), Final Viscosity (FV) and Setback Viscosity (SV) were evaluated.

Diallel analysis performed though Gardner-Eberhart Analysis II using DIALLEL-SAS05 (Zhang et al., 2005). Relative heterosis over mid-parent (MPH) and relative heterosis over better parent (heterobeltiosis) calculated as follow according to Roy (2000):

$$
\begin{gathered}
\mathrm{MPH}=\left[\frac{\overline{\mathrm{F}_{2}}-\overline{\mathrm{MP}}}{\overline{\mathrm{MP}}}\right] 100 \\
\text { Heterobeltiosis }(\mathrm{HB})=\left[\frac{\overline{\mathrm{F}_{2}}-\overline{\mathrm{BP}}}{\overline{\mathrm{BP}}}\right] 100 \\
\mathrm{SE}=\sqrt{2 \mathrm{MSE} / \mathrm{r}} \\
\mathrm{t}=\left[\left(\overline{\mathrm{F}_{2 i \mathrm{j}}}-\mathrm{MP}_{\mathrm{ij}}\right)\right] / \sqrt{\frac{3}{8}} \delta_{\mathrm{e}}^{2} \\
\mathrm{t}=\left[\left(\overline{\mathrm{F}_{2 \mathrm{ij}}}-\mathrm{BP}_{\mathrm{ij}}\right)\right] / \sqrt{\frac{1}{2}} \delta_{\mathrm{e}}^{2}
\end{gathered}
$$

Where, MSE $=$ Error mean sum of squares from RCBD ANOVA, $F_{2}=$ Mean of $F_{2}, M P=$ Mean of mid parent and $\mathrm{BP}=$ Mean of better parent.

\section{RESULTS AND DISCUSSION}

The analysis of variance of RVA parameters through Gardner-Eberhart II method (Table 2) showed that mean square of genotype was significant for all viscosity characters. Also, mean square of variety and heterosis were significant for all studied traits. Average heterosis was significant for PV, FV and SV and revealed that there was significant difference between all parents and all progenies. Variety heterosis and specific heterosis were significant for traits PV, BV, FV and SV.

The contribution of variety and heterosis effects can be calculated from the total sum of squares among diallel entries. The predominance of heterosis effects over variety effects suggests that the trait is mostly controlled by non-additive genetic effects (Laude and Carena, 2014). The contribution of variety and heterosis effects was 49.45 and $50.45 \%$, respectively, for PV. For BV, the contribution of variety and heterosis effects was 81.8 and $18.2 \%$, respectively. For FV, the contribution of variety and heterosis effects was 44.61 and $55.39 \%$, respectively. For SV, the contribution of variety and heterosis effects was 24.18 and $75.82 \%$, respectively.

Estimation of the variety effect $\left(\mathrm{v}_{\mathrm{i}}\right)$ and heterosis of varieties $\left(h_{i}\right)$ for viscosity characters (Table 3 ) revealed that the parent Line 23 had highest positive and significant value for $h_{i}$ in PV, BV and FV. Therefore, this parent had the most contribution to heterosis and the use of this parent in hybrid base breeding methods will be promising to improving these traits. The highest estimate of $\mathrm{v}_{\mathrm{i}}$ observed in the parent RI18430-46 in PV and FV, parent Deylamani for BV and parent IR50 for SV. These results indicated that these parents had the greatest effect on the variation of progenies and likewise, a greater genetic frequency of genes with additive action for these traits. Therefore, use of these parent in selection base methods to improving these traits is promising.

Results of relative heterosis through mid-parents and heterobeltiosis for viscosity characters in rice crosses (Table 4) revealed that 6 crosses had significant and negative value of $\mathrm{HB}$ and 1 cross had significant and positive value of $\mathrm{HB}$ for PV. Estimates of $\mathrm{HB}$ for PV had a range of $-14.39-13.10 \%$. crosses Deylamani $\times$ RI18447-2 and IR50 $\times$ RI18447-2 had lowest and highest HB, respectively. Results of MPH for PV showed a range of -8.70-21.50\%. Crosses RI18447-2×RI18430-46 and IR50 $\times$ Line 23 had lowest and highest MPH, respectively. These results also showed that 3 crosses had significant and negative value of MPH and 2 crosses had significant and positive value of MPH for PV. For breakdown viscosity, 8 crosses showed significant and negative HB, 4 crosses showed significant and negative MPH, 2 crosses had significant and positive HB and MPH. Estimates of HB for BV had a range of $-57.01-59.86 \%$ crosses Deylamani $\times$ Line 23 and IR50×Line 23 had lowest and highest HB, respectively. Results of MPH for BV showed a range of -38.48-66.66\% crosses Deylamani $\times$ Line 23 and IR50× Line 23 had lowest and highest MPH, respectively. In final viscosity, 3 crosses showed significant and negative $\mathrm{HB}, 2$ crosses showed significant and negative MPH, 2 crosses had significant and positive HB and 6 
Plant Sci. Res., 11 (2-4): 8-12, 2019

Table 2: Analysis of variance for viscosity characters in rice genotypes through the second method of Gardner-Eberhart

\begin{tabular}{|c|c|c|c|c|c|}
\hline \multirow[b]{2}{*}{ SOV } & \multirow[b]{2}{*}{$\mathrm{df}$} & \multicolumn{4}{|c|}{ Mean square } \\
\hline & & PV & BV & FV & SV \\
\hline$\overline{\text { Blocks }}$ & 2 & $12.29^{\text {nsec }}$ & $0.32^{\text {nsec }}$ & $9.65^{\text {nsec }}$ & $9.1^{\text {nsec }}$ \\
\hline Genotype & 20 & $1328.16^{* *}$ & $514.25^{* *}$ & $1524.84 * *$ & $110.96^{* *}$ \\
\hline Variety & 5 & $2632.15^{* *}$ & $1682.62 * *$ & $2720.94 * *$ & 107.33** \\
\hline Heterosis & 15 & $893.5^{* *}$ & $124.79 * *$ & $1126.14^{* *}$ & $112.18^{* *}$ \\
\hline Average Heterosis & 1 & 391.68* & $22.6^{\text {nsec }}$ & $981.46 * *$ & $133.11 *$ \\
\hline Variety Heterosis & 5 & $1726.99 * *$ & $111.82 * *$ & $2072.48^{* *}$ & $55.66^{*}$ \\
\hline Specific Heterosis & 9 & $486.21^{* *}$ & $143.35^{* *}$ & $616.48 * *$ & $141.25^{* *}$ \\
\hline Error & 40 & 72.77 & 29.2 & 84.95 & 20.24 \\
\hline
\end{tabular}

nsec, * and **: non-significant, significant at 5 and 1\% probability level, respectively. PV= Peak Viscosity, BV= Breakdown Viscosity, FV = Final Viscosity and SV = Setback Viscosity

Table 3: Variety effect $\left(\mathrm{v}_{\mathrm{i}}\right)$, heterosis of variety $\left(\mathrm{h}_{\mathrm{i}}\right)$ through the second method of Gardner-Eberhart

\begin{tabular}{|c|c|c|c|c|c|c|c|c|}
\hline \multirow[b]{2}{*}{ Parents } & \multicolumn{2}{|l|}{ PV } & \multicolumn{2}{|l|}{$\mathrm{BV}$} & \multicolumn{2}{|l|}{$\mathrm{FV}$} & \multicolumn{2}{|l|}{ SV } \\
\hline & $h_{i}$ & $\mathrm{v}_{\mathrm{i}}$ & $h_{\mathrm{j}}$ & $v_{\mathrm{i}}$ & $h_{i}$ & $\mathrm{v}_{\mathrm{i}}$ & $h_{i}$ & $\mathrm{v}_{\mathrm{i}}$ \\
\hline Gilaneh & $-7.88^{*}$ & $15.23^{* *}$ & $0.14^{\text {nse }}$ & $7.9^{* *}$ & $-7.59 *$ & $20.07 * *$ & $0.29^{\text {nsec }}$ & $4.85 *$ \\
\hline Daylamani & $-0.68^{\text {nsec }}$ & $13.78^{* *}$ & $-3.87^{\text {nsec }}$ & $27.93^{* *}$ & $-0.09^{\text {nsec }}$ & $12.44 *$ & $0.59^{\text {nsec }}$ & $-1.35^{\text {nsec }}$ \\
\hline IR50 & $-12.35^{* *}$ & $-18.44^{* *}$ & $-1.91^{\text {nsec }}$ & $-16.24^{* *}$ & $-18.21 * *$ & $-12.12 *$ & $-5.87 * *$ & $6.32 * *$ \\
\hline Line 23 & $32.42 * *$ & $-54.58 * *$ & $6.96 * *$ & $-18.94 * *$ & $34.02 * *$ & $-57.45^{* *}$ & $1.6^{\text {nsec }}$ & $-2.87^{\text {nsec }}$ \\
\hline RI18447-2 & $1.54^{\text {nsec }}$ & $15.48 * *$ & $2.9^{\text {nsec }}$ & $-15.8 * *$ & $4.51^{\text {nsec }}$ & $15.94 * *$ & $2.97^{\text {nsec }}$ & $0.46^{\text {nsec }}$ \\
\hline RI18430-46 & $-13.06^{* *}$ & $28.53^{* *}$ & $-4.21 *$ & $15.15^{* *}$ & $-12.64 * *$ & $21.13^{* *}$ & $0.41^{\text {nsec }}$ & $-7.4^{* *}$ \\
\hline
\end{tabular}

nsec $*$ and ${ }^{* *}$ non-significant, significant at 5 and 1\% probability level, respectively. PV = Peak Viscosity, BV $=$ Breakdown Viscosity, FV $=$ Final Viscosity and SV = Setback Viscosity

Table 4: Relative heterosis through mid-parents and heterobeltiosis for viscosity characters in rice crosses

\begin{tabular}{|c|c|c|c|c|c|c|c|c|}
\hline \multirow[b]{3}{*}{ Crosses } & \multicolumn{8}{|c|}{ Viscosity characters } \\
\hline & \multicolumn{2}{|l|}{ PV } & \multicolumn{2}{|l|}{$\mathrm{BV}$} & \multicolumn{2}{|l|}{$\mathrm{FV}$} & \multicolumn{2}{|l|}{ SV } \\
\hline & $\mathrm{HB}(\%)$ & MPH(\%) & $\mathrm{HB}(\%)$ & MPH(\%) & $\mathrm{HB}(\%)$ & MPH(\%) & $\mathrm{HB}(\%)$ & $\overline{\mathrm{MPH}(\%)}$ \\
\hline$\overline{1 \times 2^{\dagger}}$ & $2.06^{\mathrm{nsec}}$ & $3.49^{\text {nsec }}$ & $-6.27^{*}$ & $7.72^{\text {nsec }}$ & $1.21^{\mathrm{nsec}}$ & $2.43^{*}$ & $-8.94^{\text {nsec }}$ & $-3.69^{\text {nsec }}$ \\
\hline $1 \times 3$ & $-8.98^{* *}$ & $-3.42 *$ & $-23.59 *$ & $-3.77^{\text {nsec }}$ & $-6.37 *$ & $-1.79^{\text {nsec }}$ & $3.55^{\text {nsec }}$ & $6.4^{\mathrm{nsec}}$ \\
\hline $1 \times 4$ & $-1.16^{\text {nsec }}$ & $12.27 *$ & $-6.45^{\text {nsec }}$ & $21.44^{\text {nsec }}$ & $0.08^{\text {nsec }}$ & $12.73^{*}$ & $7.53^{\text {nsec }}$ & $15.16^{\text {nsec }}$ \\
\hline $1 \times 5$ & $1.76^{\text {nsec }}$ & $2.44^{\mathrm{nsec}}$ & $-13.41^{\text {nsec }}$ & $8.75^{\text {nsec }}$ & $1.72^{\text {nsec }}$ & $2.93^{* *}$ & $-0.36^{\text {nse c }}$ & $6.94^{\text {nsec }}$ \\
\hline $1 \times 6$ & $-7.86^{* *}$ & $-5.81^{* *}$ & $-4.76^{\text {nsec }}$ & $3.27^{\mathrm{nsec}}$ & $-7.17 * *$ & $-6.75^{* *}$ & $-22.45^{* *}$ & $-12.48 *$ \\
\hline $2 \times 3$ & $-6.22 *$ & $-0.74^{\text {nsec }}$ & $-40.33 * *$ & $-16.72 *$ & $-3.27^{\mathrm{nsec}}$ & $0.36^{\mathrm{nsec}}$ & $-1.18^{\text {nsec }}$ & $6.22^{\text {nsec }}$ \\
\hline $2 \times 4$ & $-14.39 *$ & $-3.03^{\text {nsec }}$ & $-57.01 * *$ & $-38.48 *$ & $-13.41^{\text {nsec }}$ & $-3.49^{\text {nsec }}$ & $-7.76^{\text {nsec }}$ & $-6.14^{\text {nsec }}$ \\
\hline $2 \times 5$ & $2.67^{\text {nsec }}$ & $4.8^{\text {nsec }}$ & $-42.55 * *$ & $-20.02^{\text {nsec }}$ & $5.97 * *$ & $7.9^{* *}$ & $17.38^{\text {nsec }}$ & 27.18* \\
\hline $2 \times 6$ & $-2.53^{\text {nsec }}$ & $-0.08^{\text {nsec }}$ & $-38.66^{*}$ & $-32.91 *$ & $-0.04^{\text {nsec }}$ & $1.24^{\mathrm{nsec}}$ & $3.64^{\text {nsec }}$ & $10.51^{\mathrm{nsec}}$ \\
\hline $3 \times 4$ & $13.1^{* *}$ & $21.59^{* *}$ & $59.86^{* *}$ & $66.66^{* *}$ & $8.85^{* *}$ & $17.32^{* *}$ & $-10.78 *$ & $-2.6^{\text {nsec }}$ \\
\hline $3 \times 5$ & $-6.52 *$ & $-0.75^{\mathrm{nsec}}$ & $7.83^{\mathrm{nsec}}$ & $8.85^{\text {nsec }}$ & $-5.37^{\mathrm{nsec}}$ & $-1.36^{\mathrm{nsec}}$ & $-12.97 *$ & $-4.38^{\text {nsec }}$ \\
\hline $3 \times 6$ & $-13.11^{* *}$ & $-5.86 *$ & $-34.74 * *$ & $-14.2^{*}$ & $-10.98 * *$ & $-6.49 *$ & $-21.18^{* *}$ & $-10^{*}$ \\
\hline $4 \times 5$ & $-7^{\text {nsec }}$ & $5.64^{\mathrm{nsec}}$ & $9.16^{*}$ & $14.62 *$ & $-3.74^{\text {nsec }}$ & $7.75^{\mathrm{nsec}}$ & $8.92^{\mathrm{nsec}}$ & $18.92 *$ \\
\hline $4 \times 6$ & $-1.95^{\text {nsec }}$ & $13.51^{* *}$ & $-16.03^{\text {nsec }}$ & $13.25^{\text {nsec }}$ & $1.03^{\text {nsec }}$ & $13.95^{* *}$ & $10.88^{\text {nsec }}$ & $16.62 * *$ \\
\hline $5 \times 6$ & $-10.64 * *$ & $-8.7 * *$ & $-24.91 * *$ & $-1.58^{\text {nsec }}$ & $-4.2^{\text {nsec }}$ & $-2.62^{\text {nsec }}$ & $29.1^{\text {nsec }}$ & $38.78^{\text {nsec }}$ \\
\hline
\end{tabular}

crosses had significant and positive MPH. Estimates of HB for FV had a range of $-13.41-8.85 \%$ crosses Deylamani $\times$ Line 23 and IR50 $\times$ Line 23 had lowest and highest HB, respectively. Results of MPH for FV showed a range of $-6.75-17.32 \%$ crosses Gilaneh $\times$ RI18430-76 and IR50 $\times$ Line 23 had lowest and highest MPH, respectively. For setback viscosity, 4 crosses showed significant and negative HB, 2 crosses showed significant and negative MPH and 3 crosses had significant and positive MPH. Estimates of HB for SV had a range of $-22.45-29.10 \%$. crosses Gilaneh×RI18430-46 and RI18447-2×RI18430-46 had lowest and highest HB, respectively. Results of
MPH for SV showed a range of $-12.48-38.78 \%$ crosses Gilaneh×R/118430-46 and RI18447-2×RI18430-46 had lowest and highest MPH, respectively.

\section{CONCLUSION}

Results of analysis of variance for viscosity characters indicated the significant difference in parents and crosses for all studied traits. The means of at least one parent has a significant difference with the mean of total parents and the mean of at least one cross has a significant difference with the mean of total crosses. Similar results 
reported by Liu (2013). Contribution of variety and heterosis effects indicated that peak viscosity controlled by additive and non-additive gene action with an approximately equal contribution. Breakdown viscosity is mostly controlled by additive gene actions due to predominance of variety effects over heterosis effects. In final viscosity non-additive gene action had a minor priority. Setback viscosity is mostly controlled by non-additive genetic effects due to predominance of heterosis effects over variety effects. Gravois and Webb (1997) reported the presence of additive effects for peak, hot paste and cool paste viscosities. Jin et al. (2004) reported additive gene action for viscosity characters.

Parent Line 23 had the most contribution to heterosis and the use of this parent in hybrid base breeding methods will be promising to improving traits PV, BV and FV.

Results of relative heterosis through mid-parents and heterobeltiosis for viscosity characters in rice crosses revealed that heterobeltiosis for peak viscosity, breakdown viscosity, final viscosity and setback viscosity had a range of $-14.39 \%$ to $13.10 \%,-57.01 \%$ to $59.86 \%$, $-13.41 \%$ to $8.85 \%$ and $-22.45 \%$ to $29.10 \%$, respectively. Results of relative heterosis through mid-parents for peak viscosity showed a range of $-8.70 \%$ to $21.50 \%$, of $-38.48 \%$ to $66.66 \%,-6.75 \%$ to $17.32 \%$ and $-12.48 \%$ to $38.78 \%$, for peak viscosity, breakdown viscosity, final viscosity and setback viscosity, respectively. Wu et al. (2002) and Liu et al. (2013) also, reported significant heterosis for viscosity characters.

\section{REFERENCES}

Allhgholipour, M., E. Farshdfar and B. Rabiei, 2014. Molecular characterization and genetic diversity analysis of different rice cultivars by microsatellite markers. Genetika, 46: 187-198.

Balindong, J.L., R.M. Ward, L. Liu, T.J. Rose and L.A. Pallas et al., 2018. Rice grain protein composition influences instrumental measures of rice cooking and eating quality. J. Cereal Sci., 79: 35-42.

Chauhan, S., Y. Ravindrababu, A.M. Patel and S. Mishra, 2016. Heterosis, combining ability and molecular characterization in fieldpea (Pisum sativum L. var arvense). J. Food Legumes, 29: 18-21.

Cozzolino, D., 2016. The use of the rapid visco analyser (RVA) in breeding and selection of cereals. J. Cereal Sci., 70: 282-290.
Falconer, D.S., 1987. [Introduction to Quantitative Genetics]. Universidade Federal de Vicosa, Vicosa, Brasil, Pages: 279 (In Portuguese).

Fitzgerald, M.A., S.R. McCouch and R.D. Hall, 2009. Not just a grain of rice: The quest for quality. Trends Plant Sci., 14: 133-139.

Fu, D., M. Xiao, A. Hayward, Y. Fu, G. Liu, G. Jiang and H. Zhang, 2014. Utilization of crop heterosis: A review. Euphytica, 197: 161-173.

Gardner, C.O. and S.A. Eberhart, 1966. Analysis and interpretation of the variety cross diallel and related populations. Biometrics, 22: 439-452.

Gravois, K.A. and B.D. Webb, 1997. Inheritance of long grain rice amylograph viscosity characteristics. Euphytica, 97: 25-29.

Griglione, A., E. Liberto, C. Cordero, D. Bressanello and C. Cagliero et al., 2015. High-quality Italian rice cultivars: Chemical indices of ageing and aroma quality. Food Chem., 172: 305-313.

Jin, Z., W. Jiang, J. Chin and H. Koh, 2004. Analysis on the combining ability of taste meter value and starch RVA properties in $\{\backslash$ sl Indica $\}$ rice. Acta Agron. Sin., 30: 1210-1214.

Koutroubas, S.D., F. Mazzini, B. Pons and D.A. Ntanos, 2004. Grain quality variation and relationships with morpho-physiological traits in rice (Oryza sativa L.) genetic resources in Europe. Field Crops Res., 86: 115-130.

Laude, T.P. and M.J. Carena, 2014. Diallel analysis among 16 maize populations adapted to the Northern US Corn Belt for grain yield and grain quality traits. Euphytica, 200: 29-44.

Liu, G.M., 2013. Heterotic analysis of starch viscosity properties in indica Hybrid rice. J. Plant Genet. Resour., 14: 434-439.

Roy, D., 2000. Plant Breeding: Analysis and Exploitation of Variation. Alpha Science International Ltd., Oxford, UK., ISBN: 9781842650066, Pages: 701.

Wu, D., S. Shen, Y. Xia and Q. Shu, 2002. Development and characterization of a low starch viscosity rice mutant. Cereal Res. Commun., 30: 301-305.

Zhang, Y., M.S. Kang and K.R. Lamkey, 2005. DIALLEL-SAS05: A comprehensive program for Griffing's and Gardner-Eberhart analysis. Agron. J., 97: 1097-1106. 